\title{
Disruption of Ephrin Signaling Associates with Disordered Axophilic Migration of the Gonadotropin-Releasing Hormone Neurons
}

\author{
John A. Gamble, ${ }^{1}$ Delicia K. Karunadasa, ${ }^{1}$ Jean-Rémi Pape,${ }^{1}$ Michael J. Skynner, ${ }^{1}$ Martin G. Todman, ${ }^{1}$ R. John Bicknell, ${ }^{1}$ \\ Jeremy P. Allen, ${ }^{1}$ and Allan E. Herbison ${ }^{1,2}$ \\ ${ }^{1}$ Neurobiology Programme, The Babraham Institute, Cambridge CB2 4AT, United Kingdom, and ${ }^{2}$ Centre for Neuroendocrinology and Department of \\ Physiology, University of Otago School of Medical Sciences, Dunedin 9001, New Zealand
}

\begin{abstract}
Ephrin signaling is involved in repulsive and attractive interactions mediating axon guidance and cell-boundary formation in the developing nervous system. As a result of a fortuitous transgene integration event, we have identified here a potential role for EphA5 in the axophilic migration of gonadotropin-releasing hormone $(\mathrm{GnRH})$ neurons from the nasal placode into the brain along ephrinexpressing vomeronasal axons. Transgene integration in the GNR23 mouse line resulted in a $26 \mathrm{~kb}$ deletion in chromosome 5, $\sim 67 \mathrm{~kb} \mathrm{3{ } ^ { \prime }}$ to Epha5. This induced a profound, region-specific upregulation of EphA5 mRNA and protein expression in the developing mouse brain. The GnRH neurons in GNR23 mice overexpressed EphA5 from embryonic day 11, whereas ephrin A3 and A5 mRNA levels in olfactory neurons were unchanged. The GnRH neurons were found to be slow in commencing their migration from the olfactory placode and also to form abnormal clusters of cells on the olfactory axons, prohibiting their migration out of the nose. As a result, adult hemizygous mice had only $40 \%$ of the normal complement of $\mathrm{GnRH}$ neurons in the brain, whereas homozygous mice had $<15 \%$. This resulted in infertility in adult female homozygous GNR23 mice, suggesting that some cases of human hypogonadotropic hypogonadism may result from ephrin-related mutations. These data provide evidence for a role of EphA-ephrin signaling in the axophilic migration of the GnRH neurons during embryogenesis.
\end{abstract}

Key words: GnRH; ephrin; migration; embryogenesis; transgenic; lhrh

\section{Introduction}

The Eph family of receptor tyrosine kinases and their ligands, the ephrins, play critical roles in cell contact-dependent signaling during nervous-system development (Flanagan and Vanderhaeghen, 1998; Frisen et al., 1999; Wilkinson, 2001; Kullander and Klein, 2002). The ephrins are divided into two groups based on their binding characteristics and structural features; EphA receptors bind principally to type A ephrins anchored to the outer plasma membrane by glycosyl phosphatidylinositol moieties, and EphB receptors bind to the transmembrane type B ephrins (Gale et al., 1996). One feature of ephrin-EphA/B signaling is its ability to exhibit bidirectional signaling such that the Eph receptor and ephrin on adjacent cells can function as both receptor and ligand (Holland et al., 1996; Knoll and Drescher, 2002). The versatility of such a signaling mechanism suggests that it is likely to be used

Received Nov. 21, 2004; revised Feb. 10, 2005; accepted Feb. 11, 2005.

This work was supported by the Biotechnology and Biological Sciences Research Council and Wellcome Trust (J.R.P. and A.E.H.). We thank Simon Andrews and John Coadwell for bioinformatics assistance, members of the Small Animal Breeding Unit at The Babraham Institute for maintaining the mice, and Mary Barter and Lucy Rowe of The Jackson Laboratory (Bar Harbor, ME) for help with genetic mapping.

Correspondence should be addressed to Prof. Allan E. Herbison, Centre for Neuroendocrinology and Department of Physiology, University of Otago School of Medical Sciences, P.0. Box 913, Dunedin 9001, New Zealand. E-mail: allan.herbison@stonebow.otago.ac.nz.

DOI:10.1523/JNEUROSCI.4759-04.2005

Copyright $\odot 2005$ Society for Neuroscience $\quad$ 0270-6474/05/253142-09\$15.00/0 widely in development of the nervous system. Characterized initially as a signaling system mediating repulsion in axon guidance and boundary formation, recent studies have extended ephrin actions to also include attractive and adhesive roles in neural development (Wilkinson, 2001; Holmberg and Frisen, 2002). Although the precise nature of these adhesive and repulsive properties is not yet established, recent work examining ephrin signaling in axon guidance suggests that an Eph-ephrin interaction may be repulsive or attractive depending on ligand concentration and the local microenvironment (Weinl et al., 2003; Hansen et al., 2004).

One of the most dramatic examples of cell migration during development of the mammalian brain is that provided by the gonadotropin-releasing hormone $(\mathrm{GnRH})$ neurons. These cells are born outside of the brain and migrate from the nasal placode along vomeronasal nerve axon bundles to enter the forebrain during embryogenesis (Schwanzel-Fukuda and Pfaff, 1989; Wray et al., 1989). The mechanisms underlying this long-range axophilic migration remain mostly unknown (Wray, 2002). Once established in the hypothalamus, the $\mathrm{GnRH}$ neurons represent the central neuronal phenotype controlling the reproductive axis. Consequently, defects in GnRH neuronal migration in humans result in infertility (Hardelin, 2001; MacColl et al., 2002a).

In the course of a program of work involving the production of $\mathrm{GnRH}$ promoter transgenic mice, we fortuitously created a line 
of mice (GNR23) in which the numbers of GnRH neurons in the hypothalamus were dramatically reduced. We demonstrate here that insertion of the GnRH promoter construct in the GNR23 line resulted in a $26 \mathrm{~kb}$ deletion of sequence $\sim 67 \mathrm{~kb} 3^{\prime}$ to the Epha5 gene on chromosome 5. This induced massive, regionspecific upregulation of EphA5 expression in the developing brain, including the GnRH neurons, and resulted in their defective migration along vomeronasal nerve axons into the brain. These observations suggest that the roles of ephrin signaling during neuronal development can be extended to include that of axophilic $\mathrm{GnRH}$ neuron migration.

\section{Materials and Methods}

GNR23 mice. All mice were bred and housed at The Babraham Institute according to UK Home Office requirements under Project License 80/ 1475, and experiments were approved by The Babraham Institute Animal Welfare and Ethics Committee. Mice were maintained under $12 \mathrm{~h}$ lighting conditions (lights on at 7:00 A.M.) with food and water available ad libitum. The GNR23 mouse was created using a transgene construct that contains the Escherichia coli nitroreductase (NR) gene (Isles et al., 2001) under the transcriptional control of mouse GnRH regulatory sequences. HindIII and BamHI sites were introduced $5^{\prime}$ and $3^{\prime}$, respectively, relative to the NR open reading frame. The resulting $680 \mathrm{bp}$ HindIII-BamHI fragment was then ligated to 6383 bp of upstream GnRH sequence, including GnRH exon I, intron A, and the first 7 bp of exon II (Pape et al., 1999). The GnRH-NR expression cassette was excised from the vector backbone using Eco47III and NotI. Transgenic mice were produced by pronuclear microinjection of day 1 embryos and subsequent embryo transfer to pseudopregnant dams, according to standard procedures. GNR23 mice were genotyped in a multiplex PCR assay using two sets of PCR primers. NR-F (5'-CATTCCACTAAGGCATTTGA TGC-3') and NR-R (5' ${ }^{\prime}$ TTCAGACCAAATTCTGCATCGAG-3') amplify a 506 bp fragment from the E. coli NR gene. GNR23-GT-F3 (5' TGTATTAACAAAACCCAGCCATACC-3') and GNR23-GT-R2 (5'CTTCCACTCTCCCTCTGTATCTTCC-3') amplify a 196 bp fragment from a region of genomic DNA known to be deleted in 2300 mice as a consequence of transgene integration. Primers were used at a final concentration of $0.25 \mu \mathrm{M}(\mathrm{NR}-\mathrm{F}+\mathrm{R})$ and $1 \mu \mathrm{M}(2300-\mathrm{GT}-\mathrm{F} 3+\mathrm{R} 2)$ and annealed at $60^{\circ} \mathrm{C}$.

GNR23 genomic library screening. Genomic DNA was prepared from a heterozygous GNR23 mouse liver, partially digested with Sau3A, and cloned into Lambda Fix II (Stratagene, La Jolla, CA). The library was screened with a $200 \mathrm{bp}$ probe from mouse GnRH intron A, randomprime labeled (Amersham Biosciences, Piscataway, NJ) with $\left[\alpha^{-}{ }^{32} \mathrm{P}\right]$ deoxyCTP. All nucleic acid hybridization experiments were performed according to standard protocols.

Vectorette PCR. Vectorette PCR was performed using the Vectorette II Starter Pack (DN-16-020A; Sigma, St. Louis, MO) according to the manufacturer's instructions. Briefly, genomic DNA was digested with BamHI, ClaI, EcoRI, HindIII, or SmaI. Double-stranded DNA linkers bearing the appropriate complementary termini were then ligated to the digested DNA, and nested PCR was performed using primers based both in the NR transgene and in the Vectorette linker. Amplified products were cloned into pGEM-T (Promega, Madison, WI) and sequenced.

Backcross mapping. Genomic DNA from C57BL/6J and Mus spretus mice was obtained from The Jackson Laboratory (Bar Harbor, ME), and scorable polymorphisms were identified in DNA sequences flanking the site of transgene integration. (1) The $3^{\prime}$ flank: PCR primers $2300 / 3^{\prime}-\mathrm{F} 8$ (5'-GGCCATATATCCAACTACTGTGC-3') and 2300/3'-R8 (5' ${ }^{\prime}$-TTA CCTGCAGACCCATCTCC-3') amplify a 213 bp fragment (located 1277 bp 3' of the transgene) from C57BL/6J DNA, whereas amplification from $M$. spretus generates a smaller fragment, as resolved on a $3 \%$ agarose gel. (2) The 5' flank: PCR primers 2300/5'-F5 (5'-AGATTTGACACACT GCACATAAGATTG-3') and 2300/5'-R5 (5'-TTATTATAAACCTCGA TTCCCACTCAG-3') amplify a 489 bp fragment (lying 454 bp $5^{\prime}$ of the transgene) that contains a PvuII site in C57BL/6J but not in M. spretus DNA. The haplotypes of The Jackson Laboratory interspecific backcross panels TJL BSB [(C57BL/6J $\times$ M. spretus $\left.) \mathrm{F}_{1} \times \mathrm{C} 57 \mathrm{BL} / 6 \mathrm{~J}\right]$ and TJL BSS
[(C57BL/6J $\times$ SPRET/Ei) $F_{1} \times$ SPRET/Ei] were then determined (Rowe et al., 1994). Haplotype data were submitted to The Jackson Laboratory for the determination of the strain distribution pattern and chromosomal location of markers (http://www.jax.org/resources/documents/ cmdata).

Immunocytochemistry and in situ hybridization. The immunocytochemical analysis of embryonic and postnatal mouse brain tissue was undertaken using protocols published previously (Skynner et al., 1999; Simonian and Herbison, 2001b), using antibodies raised against GnRH [1:5000-1:20,000, LR1; a gift from R. Benoit (Montreal Hospital, Montreal, Quebec, Canada); or 1:10,000, catalog \#20075; DiaSorin (Stillwater, $\mathrm{MN})$ ], tyrosine hydroxylase (1:4000, MAB318; Chemicon, Temecula, CA), somatostatin (1:3000; a gift from F. Vandesande, Zoological Institute, Leuven, Belgium), choline acetyltransferase (1:5000, AB144; Chemicon), peripherin (1:4000, AB1530; Chemicon), polysialated neural cell adhesion molecule (PSA-NCAM) (1:1000; a gift from G. Rougon, Institute de Biologie du Development, Marseille, France), and the extracellular domain of EphA5 (1:100, E3654; Sigma-Aldrich, Poole, UK). EphA5 immunofluorescence was undertaken by incubating in the goat affinity-purified EphA5 antibody, followed by biotinylated anti-goat IgGs (Vector Laboratories, Peterborough, UK), Vector Elite ABC reagent, and tyramide-Alexa 594 (Molecular Probes, Eugene, OR).

The quantitative analysis of GnRH immunostaining was undertaken as reported previously (Pape et al., 1999; Simonian and Herbison, 2001a). In postnatal brains, coronal sections containing the medial septum (MS), preoptic area (POA), and anterior hypothalamic area (AHA), represented by plates 21-34 of the mouse brain atlas of Franklin and Paxinos (1997) and depicted in Figure 1G, were selected. Two forms of analysis were undertaken; in the first, the numbers of $\mathrm{GnRH}$ neurons were estimated in each of the three areas by analyzing three sections from each area and providing mean GnRH neuron/section values. In the second type of analysis, every second coronal section through the MS, POA, and AHA was immunostained for GnRH, and every GnRH neuron was counted. This method provided a more reliable method of estimating the total GnRH neuron population. Embryonic brains were analyzed by determining the location of GnRH neurons in three midsagittal sections from each embryo. Any GnRH neuron located within $50 \mu \mathrm{m}$ of the vomeronasal organ was counted as being within, or adjacent to, the vomeronasal organ. Cell counts are given per section.

In situ hybridization was undertaken, also as published previously (Simonian and Herbison, 2001b), using 40-48 $\mathrm{mer}^{35}$ S-labeled oligonucleotide probes directed against murine GnRH-I (CTCTTGGAAAGACTCAACCAAGTGTTCAGTGTTTCTCTTTCCCCC), EphA5 (kinase domain, CACCATCCCGTTTACCATCTGCACCTTCATCTCTT; transmembrane domain, GATGGGAATCTGGCTTTGATCATTAGATGCTGCAA; extracellular domain, CTTCACCAATCTCTTCCCACCCGTTCTTT), ephrin A3 (CCTGATCCACTACGGTGCCTGTCCTAGAGAGT and GACAAAGAGGGAGGGCACCAAGGGTCAGGGAAA), and ephrin A5 (TGAACACACACATCCAGAGCACCAGAAAGAGCA and CATCAATGTGGTAGTCACCCCTCTGGAATCTGG). Hybridization specificity was determined by comparison between probes and by competition experiments in which a radiolabeled probe was hybridized in the presence of a 25 -fold excess of "cold" unlabeled oligonucleotide. EphA5 mRNA expression was quantified using an image analysis system (AIS; Imaging Research, St. Catharines, Ontario, Canada). Only silver grain counts of more than five times that of corresponding areas in "cold oligo" control slides were considered to represent positive hybridization.

\section{Results}

\section{Phenotypic characterization of the GNR23 mouse}

Adult GNR23 mice

The NR gene can be used for conditional ablation strategies by driving expression of the NR protein in specific cell phenotypes and administering the appropriate prodrug (Isles et al., 2001). In making a GnRH promoter-NR transgenic mouse, we produced six founder mice. Of these, only one (GNR662) was identified as expressing the NR protein by Western blot. However, the initial screening of untreated, hemizygous offspring from these lines 
revealed that adult mice of the GNR23 line had reduced numbers $\mathrm{GnRH}$-immunoreactive neurons in the brain (Fig. 1A-C). Cell counts showed that adult male hemizygous GNR23 mice had only $\sim 37 \%$ of the normal number of GnRH neurons [controls, $n=7 ; 10.9 \pm 0.4,25.6 \pm 0.5$, and $7.2 \pm 0.4 \mathrm{GnRH}$ neurons per section in the MS, POA, and AHA, respectively, compared with $6.9 \pm 0.4,7.4 \pm 0.3$, and $2.0 \pm 0.2$ in hemizygous GNR23 mice $(n=8)$ ]. In situ hybridization revealed an identical reduction, with only $36 \%$ of the normal numbers of GnRH mRNAexpressing cells remaining in the brains of hemizygous adult male GNR23 mice $(n=5)$ compared with controls $(n=5)$. The topography and number of GnRH neurons in the other five lines, including hemizygous NR-expressing GNR662 mice (Fig. 1B), were all normal, indicating that NR without the prodrug is benign (Isles et al., 2001).

Homozygous (hmz) GNR23 mice were found to display an even more profound depletion, with only $\sim 16 \%$ ( $n=5-6$, each sex) of normal GnRH cell numbers present in the brain (Fig. $1 D, G)$. Cell counts of every second section through the MS, POA, and AHA showed that adult male hmz GNR23 mice $(n=5)$ had a total of $21.8 \pm 2.8,13.8 \pm 2.5$, and $4.0 \pm 0.7 \mathrm{GnRH}$ neurons in these areas, respectively, compared with $63.4 \pm 4.6,116.2 \pm 4.5$, and $69.4 \pm 7.3 \mathrm{GnRH}$ neurons in wild-type adult male mice $(n=$ 5). This showed that hmz GNR23 mice had only 34,12 , and $6 \%$ of the wild-type complement of GnRH neurons in the MS, POA, and AHA (Fig. 1G). The same deficit was found in adult female hmz GNR23 mice $(n=6)$ with only 31,11 , and $5 \%$ of the normal numbers of GnRH neurons in the MS, POA, and AHA (controls, $n=6$ ). In both sexes and in hemizygous as well as hmz GNR23 mice, there was clear trend for GnRH neurons located caudalmost in the brain to display the greatest deficit in cell numbers (Fig. 1G). These cell counts estimated that adult male and female hmz GNR23 mice had a total of $78 \pm 3 \mathrm{GnRH}$ neurons in the forebrain compared with $502 \pm 17$ in the MS, POA, and AHA of wild-type mice. The axons of GnRH neurons in GNR23 mice innervated the organum vasculosum of the lamina terminalis and median eminence, their two principal targets, in a normal, albeit hugely reduced, manner (Fig. $1 C-F$ ).

Gross and histological analysis of the brains of GNR23 mice appeared normal. Quantitative immunocytochemical evaluations of other neuroendocrine phenotypes (dopamine neurons in the arcuate nucleus and periventricular somatostatin neurons) and neuronal cell populations located in the same locations as the GnRH neurons (medial septal acetylcholine neurons and rostral POA dopamine neurons) revealed no abnormalities in cell number or distribution (data not shown). These data suggested that a selective, allele dose-dependent alteration of the GnRH neuronal phenotype existed in the GNR23 mouse and that this was unrelated to the NR protein itself.

Embryonic GNR23 mice

In the mouse, GnRH neurons are first visible in and around the developing vomeronasal organ/nasal placode on embryonic day 11 (E11) and migrate into the brain over the following $6 \mathrm{~d}$ (Wray, 2002). At these times, they are visible as streams of neurons on bilateral olfactory axon tracks moving through the nose toward the cribriform plate to enter the forebrain (Fig. 2A, B). Immunocytochemical and in situ hybridization analyses of GnRH and GnRH mRNA expression at E11.5, E12.5, and E14.5 revealed that GnRH neurons in GNR23 mice were slow in beginning their migratory journey from the nasal placode and that, once started, most then formed into large atypical spherical clusters along the nasal migratory route (Fig. 2C-F). In E11.5 wild-type embryos,
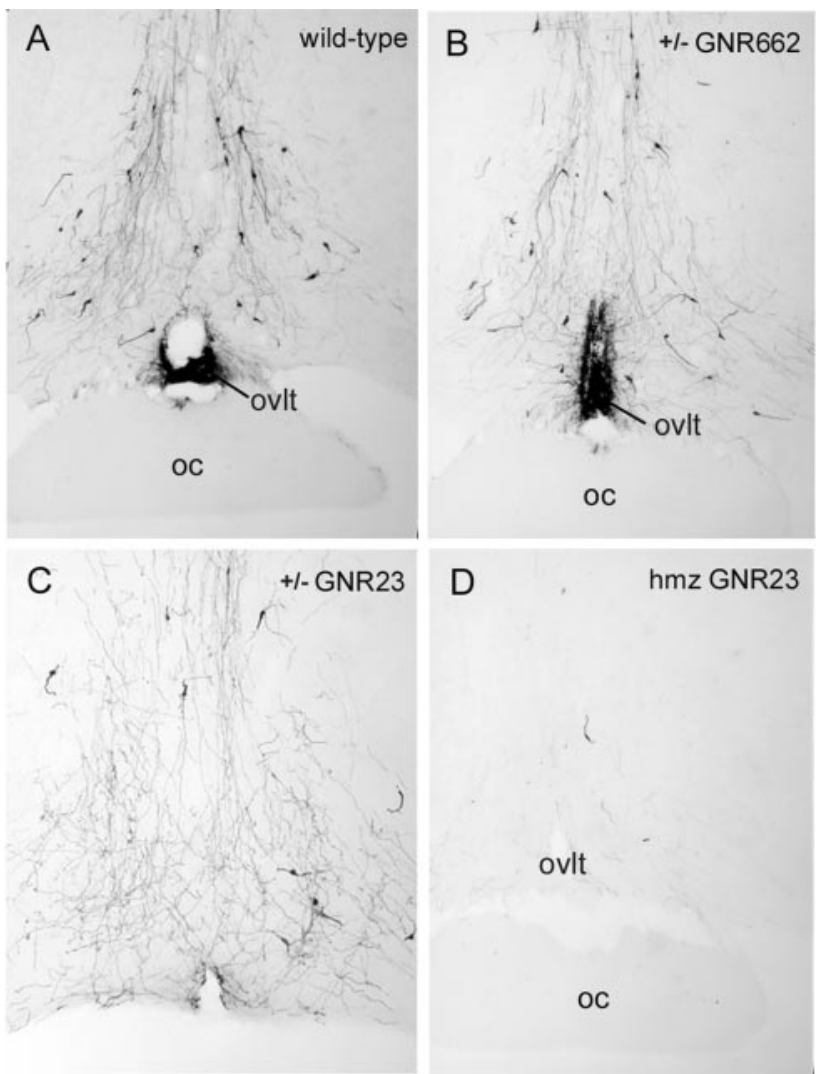

hmz GNR23
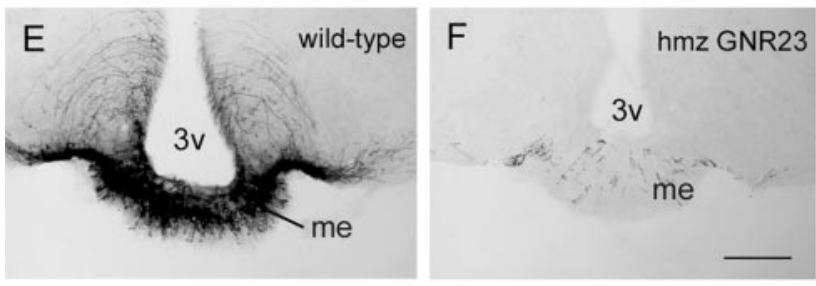

\section{G}

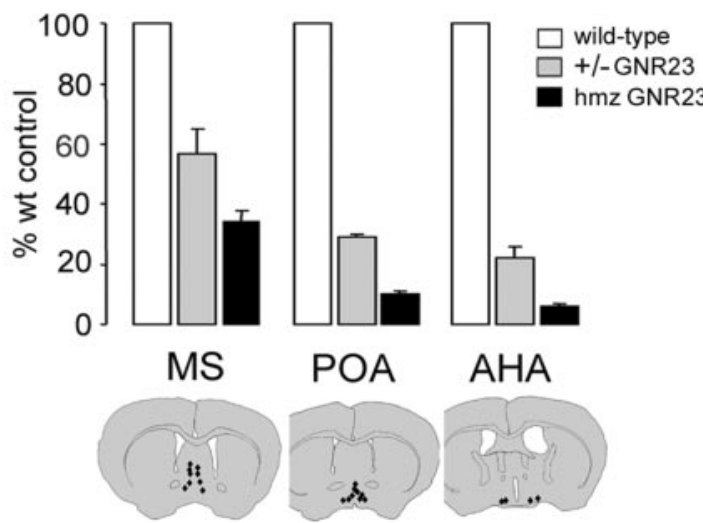

Figure 1. Major loss of GnRH neurons in the brain of adult GNR23 mice. $A$, GnRHimmunoreactive cell bodies are located principally within the POA of the wild-type mouse brain, and normal numbers of GnRH neurons are found in hemizygous GNR662 mice that express NR (B). Only $36 \%$ of the normal GnRH population is observed in hemizygous GNR23 mice ( $\boldsymbol{C}$ ), and this is reduced to $15 \%$ in hmz animals (D). The principal fiber projections of the $\mathrm{GnRH}$ neurons are to the organum vasculosum of the lamina terminalis (ovlt; $\boldsymbol{A}, \boldsymbol{B}$ ) and median eminence (me, $\boldsymbol{E}$ ), and both regions show a massive loss of $\mathrm{GnRH}$ fibers in GNR23 mice $(\boldsymbol{C}, \boldsymbol{D}, \boldsymbol{F})$. $\mathbf{G}$, Quantitative analysis of $\mathrm{GnRH}$ neuron number at three coronal levels of the forebrain (MS, $P O A$, and $A H A$ ) of adult male mice ( $n=5-8$ each genotype). Black dots indicate locations of $\mathrm{GnRH}$ neuron cell bodies at the three coronal levels. Histograms show the percentage of wildtype (wt) GnRH neuron number found at each level in the hemizygous (gray) and hmz (black) GNR23 adult male mice. Note that the deficit in GnRH neuron numbers is greater the farther caudal the $\mathrm{GnRH}$ neurons must migrate within the brain. oc, 0ptic chiasm; $3 \mathrm{v}$, third ventricle. Scale bar: (in $\boldsymbol{F}) \boldsymbol{A}-\boldsymbol{F}, 200 \mu \mathrm{m}$. 

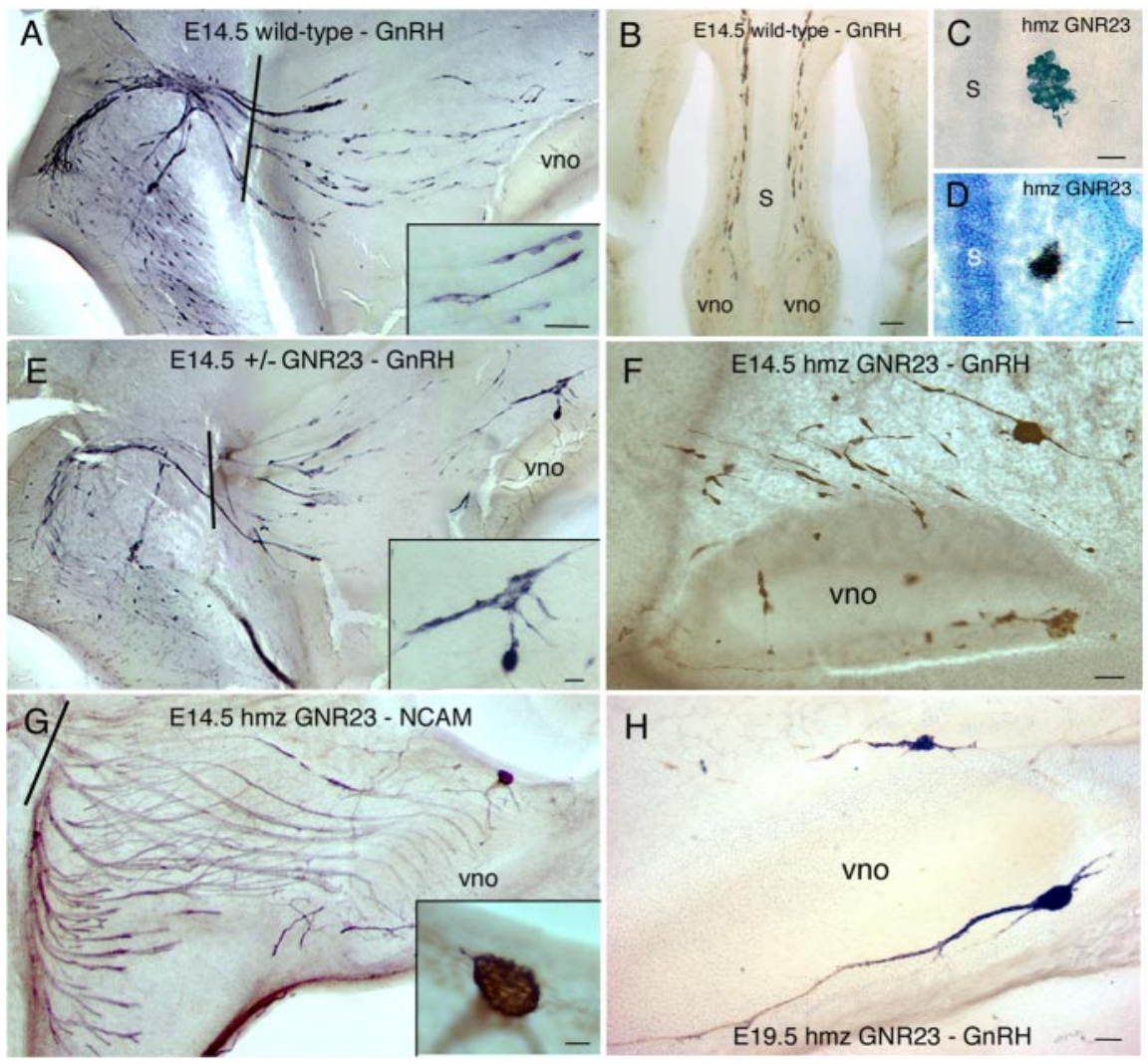

Figure 2. GnRH neurons fail to migrate into the brain of GNR23 mice during embryogenesis. The GnRH neurons migrate from the vomeronasal organ (vno) into the brain along olfactory axons as individual streams of cells that converge to cross through the cribriform plate (vertical line) and enter the brain. All sagittal sections $(\boldsymbol{A}, \boldsymbol{E}-\boldsymbol{H})$ are orientated in the same manner with brain to left. $\boldsymbol{A}$, Wild-type E14.5 midsagittal section stained for $\mathrm{GnRH}$. The inset shows a high-power view of normally migrating $\mathrm{GnRH}$ neurons. $\boldsymbol{B}$, Coronal view of a wild-type E14.5 embryo stained for $\mathrm{GnRH}$ showing streams of $\mathrm{GnRH}$ neurons running up either side of the nasal septum (S) from the bilateral vno to the brain (top). C, Same orientation but a higher-power view of a spherical cluster of GnRH-immunoreactive neurons adjacent to the nasal septum (S) in an hmz GNR23 E14.5 embryo. D, Similar view of a cluster of GnRH mRNA-expressing cells (black silver grains) adjacent to the nasal septum in an hmz GNR23 E14.5 embry0. E, Lower-power, midsagittal view of GnRH-immunoreactive neurons migrating into the brain of a hemizygous GNR23 E14.5 embryo. Note the abnormal clustering of $\mathrm{GnRH}$ neurons along the streams and immediately above the vno (inset). $\boldsymbol{F}$, Medium power midsagittal view of GnRH immunoreactivity around the vno of an E14.5 hmz GNR23 embryo. Note the spherical cluster of GnRH neurons with leading and trailing processes above the vno and a farther cluster at the ventral edge of the vno. Other neurons appear to be migrating normally. G, Midsagittal view of PSA-NCAM staining in an E14.5 hmz GNR23 embryo showing a normal axon scaffold from the vno into the brain. Note also the cluster of NCAM-immunoreactive cells immediately dorsal to the vno (inset) that was shown on a consecutive section to be a cluster of $\mathrm{GnRH}$ neurons. $\boldsymbol{H}$, Medium power, midsagittal view of $\mathrm{GnRH}$ immunostaining in the nose of an E19.5 hmz GNR23 embryo showing two clusters of multiple GnRH neurons with leading and trailing processes on either side of the vno. Scale bars: $\boldsymbol{A}, \boldsymbol{C}-\boldsymbol{G}, 50 \mu \mathrm{m} ; \boldsymbol{B}, \boldsymbol{H}, 100 \mu \mathrm{m}$.

approximately one-half $(52 \pm 3 \%)$ of the GnRH-immunoreactive neurons were found to be located within and adjacent to the nasal placode, with the other half $(48 \pm 3 \%)$ having commenced their migration ( $28 \pm 4 \mathrm{GnRH}$ neurons in the nose per parasagittal section; $n=5$ ). In contrast, in E11.5 hemizygous GNR23 embryos, $80 \pm 3 \%$ of GnRH neurons were located around the placode, with only $20 \pm 3 \%$ outside ( $29 \pm 3 \mathrm{GnRH}$ neurons per parasagittal section; $n=5)$. In E11.5 hemizygous and hmz GNR23 embryos, GnRH neurons were also observed to form clusters of up to 5-10 $\mathrm{GnRH}$ neurons. From E12.5 onward, these atypical clusters of GnRH neurons in GNR23 mice were much larger and existed as large spherical masses of up to $100 \mathrm{GnRH}$ cell bodies with bundles of leading and trailing GnRH axons in the correct orientation (Fig. $2 C-F$ ). Immunocytochemistry and in situ hybridization (Fig. 2D) revealed that spherical clusters of GnRH neurons existed all along the migratory route within the nose but that the great majority $(>80 \%)$ were adjacent to or within $200 \mu \mathrm{m}$ of the vomeronasal organ

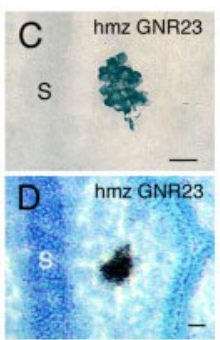

(Fig. 2E-H). However, even in hmz GNR23 embryos, some GnRH neurons exhibited an apparently normal migratory appearance (Fig. $2 F$ ).

Evaluation of the olfactory/vomeronasal axons, on which the GnRH neurons migrate, with both peripherin (Wray et al., 1994) and PSA-NCAM (SchwanzelFukuda et al., 1992; Yoshida et al., 1999) immunocytochemistry revealed no abnormalities in GNR23 mice (Fig. 2G), suggesting that the axonal scaffold for the migration was intact. However, large spherical clusters of NCAM-positive cells identical to the GnRH clusters were observed in GNR23 mice (Fig. 2G, inset). The examination of another long-range migratory pathway, the rostral migratory stream, with PSA-NCAM revealed no differences between adult GNR23 and wild-type siblings (data not shown).

Examination of E19.5 and postnatal day 1 mice demonstrated the continued presence of GnRH clusters in the nose of GNR23 mice (Fig. 2H). The extensive clustering of GnRH neurons made accurate analysis of total GnRH neuron cell number impossible. However, E11.5 hemizygous GNR23 mice exhibited the least clustering, and $\mathrm{GnRH}$ neuron counts in the nose (three parasagittal sections per embryo; $n=5$ embryos per group) revealed equivalent numbers of GnRH neurons in wild-type ( $82 \pm 11$ neurons $)$ and hemizygous GNR23 $(86 \pm 10)$ embryos. Together, these data indicate that the respective 60 and $85 \%$ depletion of $\mathrm{GnRH}$ neuron numbers in the brains of postnatal hemizygous and hmz GNR23 mice results from the failed migration of $\mathrm{GnRH}$ neurons into the forebrain. The numbers of GnRH neurons born in the nasal placode are not different in GNR23 mice, but the great majority fail to complete their migration into the brain as a result of extensive clustering along the vomeronasal axon pathways. As a result, adult hmz GNR23 female mice exhibit marked infertility. Seven of $14 \mathrm{hmz}$ adult GNR23 female mice tested were infertile, with the rest usually providing only a single litter. This resulted in a litter rate of $0.6 \pm 0.2$ litters per female for the first 4 months of breeding for hmz GNR23 mice compared with $4.6 \pm 0.5$ litters from hemizygous GNR23 mice $(n=5)$ and $4.7 \pm 0.5$ litters from wild-type adult female mice $(n=7)$.

\section{Genotypic characterization of the GNR23 mouse}

Flanking sequence

The GNR23 mouse was generated by conventional pronuclear (C57BL/6J $\times$ CBA/Ca) injection of a $\sim 7 \mathrm{~kb}$ GnRH-NR construct [5.8 $\mathrm{kb}$ of 5' GnRH sequence (Pape et al., 1999) linked to NR] (Fig. 3A). Regions of genomic DNA flanking the integrated transgene in GNR23 mice were isolated by a combination of Vectorette PCR and genomic library screening. Using a gene-specific primer based in the unique NR gene and Vectorette PCR, we 
initially isolated two overlapping clones of 1101 bp (pVec-BglII) and 1524 bp (pVecEcoRI), representing genomic DNA immediately $3^{\prime}$ of the transgene (Fig. $3 B$ ). To identify additional flanking DNA, we constructed and screened a GNR23 genomic library. We obtained two clones that further extended the $3^{\prime}$ flank (p11.1 and p13.1) and also three overlapping clones containing sequence directly flanking the $5^{\prime}$ end of the transgene insertion site (p1.2, p1.10, and p2.1) (Fig. 3B). Combined, these clones provided almost $4 \mathrm{~kb}$ of $3^{\prime}$ and $5 \mathrm{~kb}$ of $5^{\prime}$ flanking sequence, and indicated a single transgene integration site.

\section{Genomic mapping}

Sequence analysis of both the $3^{\prime}$ and $5^{\prime}$ flanking clones showed that they contained multiple LINE-1 repeats in addition to nonrepeat sequences. Mapping of the latter within the Ensembl C57BL/6J mouse genome database (http://www.ensembl.org/Mus_musculus/) showed that the transgene had inserted in reverse orientation into chromosome 5 (Fig. $3 B$ ). D5Mit markers $(7,18,205$, and 312) that are polymorphic between C57BL/6J and CBA/Ca and close to the site of transgene integration were typed and showed that the transgene had integrated into the C57BL/6J allele (data not shown), validating our use of the Ensembl database to characterize the GNR23 allele. As an independent assessment of the transgene integration site, we typed two interspecific backcross panels using polymorphic markers, D5Bne1 and D5Bne2, that we identified within the $3^{\prime}$ and $5^{\prime}$ flanking sequences (Fig. $3 B$ ). In both crosses, D5Bne1 and D5Bne2 segregated with D5Mit40 and D5Mit414 (http://www. jax.org/resources/documents/cmdata) (Fig. $3 C)$. With respect to chromosome 5 genomic sequence, D5Mit40 and D5Mit414 are tightly linked to the transgene flanking sequence (Fig. 3C), confirming the location of transgene integration.

Alignment of both transgene flanking sequences with the corresponding genomic DNA (Ensembl version 16.30.01) demonstrated that the $5^{\prime}$ and $3^{\prime}$ flank, were separated by 25,685 bp ( $5^{\prime}$ flank, base pair 83194895 ; 3' flank, base pair 83169210), suggesting that transgene integration had been accompanied by deletion of this fragment. The presence and extent of this deletion was confirmed by PCR and Southern blot. Individual sets of PCR primers were designed in nonrepeat sequences, at intervals along the length of the putative $25.7 \mathrm{~kb}$ deletion and its flanking sequence (Fig. 3D). Primers directed at $5^{\prime}$ and $3^{\prime}$ flanking regions of the transgene insertion site amplified product from both wild-type and GNR23 genomic DNA, whereas primers directed against the deleted region were only successful in

E.
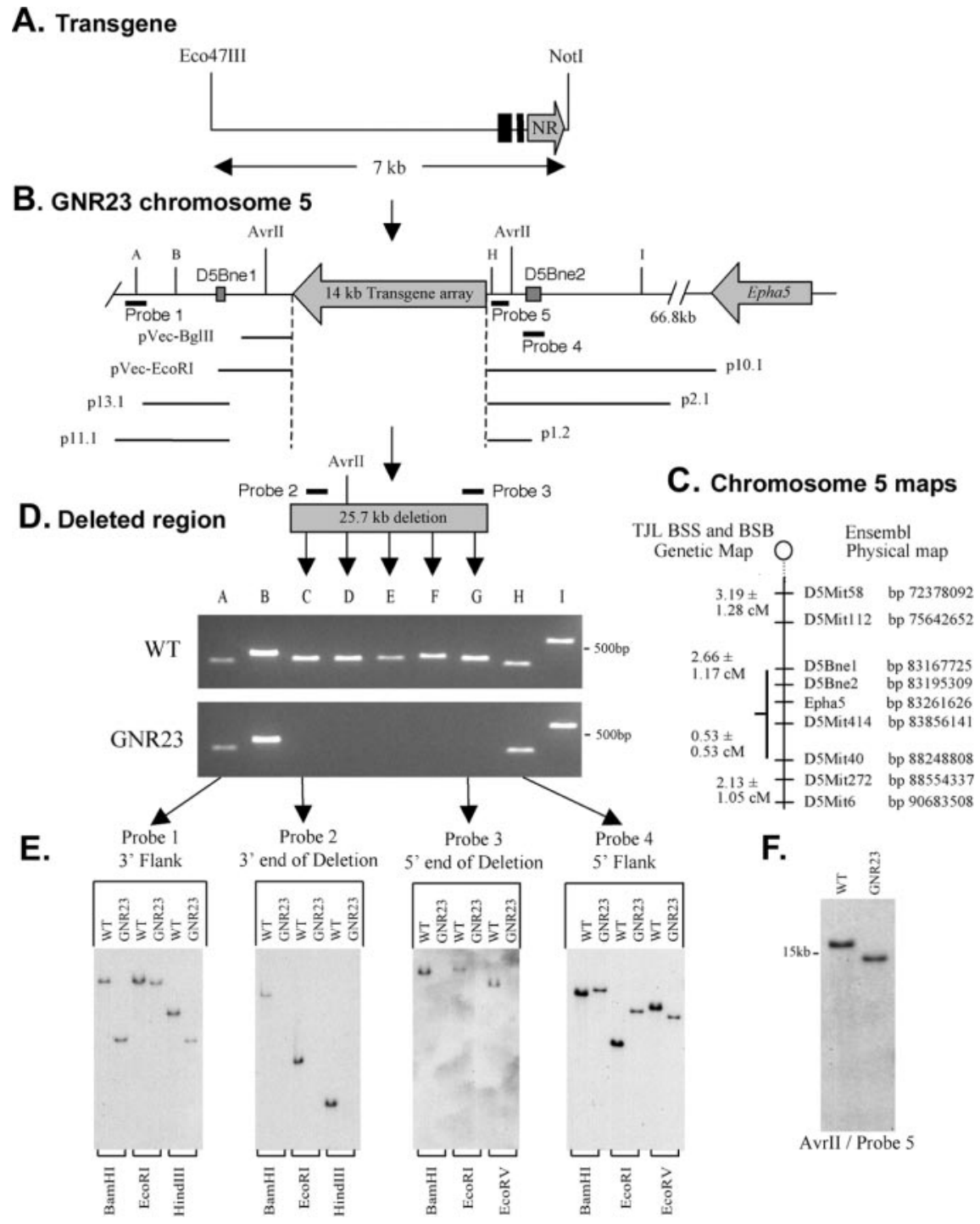

Figure 3. Genomic characterization of the GNR23 allele, showing integration of $\sim 14 \mathrm{~kb}$ of transgene-specific DNA into the antisense strand of chromosome 5 and concomitant deletion of $25.7 \mathrm{~kb}$ of wild-type DNA. The $7 \mathrm{~kb} \mathrm{GnRH}-\mathrm{NR}$ transgene $(\boldsymbol{A})$ and the integration site on chromosome $5 \sim 67 \mathrm{~kb}$ proximal to Epha5 $(\boldsymbol{B})$. The $5^{\prime}-3^{\prime}$ orientation of the transgene array and the Epha5 open reading frames are indicated by arrows. Positions of flanking DNA clones isolated by Vectorette PCR (pVec-Bg/ll, pVec-EcoRI) and by library screening (p13.1, p11.1, p10.1, p2.1, p1.2), and the markers D5Bne1 and D5Bne2 are shown relative to the transgene. Fragments of DNA are not drawn to scale. C, Markers D5Bne1 and D5Bne2, which flank the site of transgene integration, were mapped to chromosome 5 on two separate interspecific backcross panels, TJL BSS and TJL BSB. A combined BSS/BSB map is shown, centromere to top, with loci linked to D5Bne1 and D5Bne2. Genetic distances (CM) between markers are shown \pm SEM. Epha 5 was not mapped in either backcross panel. The Ensembl chromosome 5 physical map shows these genetic markers ordered according to their positions in the Ensembl mouse genome database (version 16.30.01). The base pair (bp) number indicates the location of the $5^{\prime}$ end of each marker. This illustrates the relative position of Epha5 with respect to the backcross mapping data. $\boldsymbol{D}$, The $25.7 \mathrm{~kb}$ deleted region predicted by in silico analysis using the Ensembl database was characterized using PCR assays $A-I$. The positions of $A, B, H$, and $\boldsymbol{I}$ are depicted in $\boldsymbol{B}$. Amplification from GNR23 DNA is successful from PCR assays that flank the deletion, $\boldsymbol{A}, \boldsymbol{B}, \boldsymbol{H}$, and $\boldsymbol{I}$, but is unsuccessful from assays $\boldsymbol{C}-\boldsymbol{G}$ internal to the deletion. $\boldsymbol{E}$, The deleted region was further characterized by Southern blot using probes located adjacent to (probes 1 and $4, \boldsymbol{B}$ ) and within (probes 2 and $3, \boldsymbol{D}$ ) the $5^{\prime}$ and 3 ' limit of the deletion. Fragments of the predicted size were observed in both wild-type and GNR23 using probes 1 and 4 . Probes 2 and 3 detected predicted fragments from wild type, but failed to detect any fragments from GNR23. $\boldsymbol{F}$, Southern blot showing approximate size of the transgene insertion. Sites for Avrll are not contained within the transgene, but are present immediately adjacent to the transgene array $(\boldsymbol{B})$, and located $16.8 \mathrm{~kb}$ from the distal end of the deletion (D). Probe 5 (shown in $\boldsymbol{B}$ ) identifies fragments of $\sim 17 \mathrm{~kb}$ for wild-type $(57 \mathrm{BL} / 6$ ) and $14 \mathrm{~kb}$ for GNR23. WT, Wild type.

amplification from wild-type DNA (Fig. 3D). The $5^{\prime}$ and $3^{\prime}$ boundaries of the deletion were further confirmed by Southern blot analysis using probes situated internal to and on either side of the putative deletion (Fig. 3E). This confirmed that the $25.7 \mathrm{~kb}$ region of genomic DNA has been deleted from the GNR23 genome and not translocated/rearranged to another part of the genome. The deleted 
DNA was highly repetitive (48.6\%), and no sequence similarity to any known gene was found. Excision of the transgene array using AvrII showed that the deleted DNA had been replaced by $\sim 14 \mathrm{~kb}$ of transgene-specific sequences (Fig. $3 F$ ).

Ensembl genomic sequence analysis showed that the nearest gene to the site of transgene integration was Epha5, which lies on the reverse strand of DNA, $\sim 66.8 \mathrm{~kb}$ distal to the transgene (Fig. $3 B)$. No other genes have been located, or are predicted, within $500 \mathrm{~kb}$ either side of the transgene insertion site. This raised the possibility that Epha5 gene expression may be altered.

\section{EphA5 and ephrin-A expression in the GNR23 mouse \\ EphA5 expression}

In situ hybridization experiments in E11.5, E12.5, and E14.5 embryos revealed abnormal EphA5 mRNA expression throughout the brain of GNR23 mice. As has been noted previously in wildtype embryos (Zhang et al., 1996), EphA5 mRNA was expressed in a heterogenous manner in the developing mouse brain, with the highest levels of EphA5 mRNA detected in the developing neocortex, globus pallidus, and hippocampus. Significant 1.5- to 40-fold increases in EphA5 mRNA expression were detected in the developing lateral septum, globus pallidus, hippocampus, and mediobasal hypothalamus of E14.5 hmz GNR23 embryos (Fig. $4 B, E)$ ( $n=4$ each genotype). No change was detected in the olfactory bulb and cortex (Fig. $4 A, B, E$ ). At postnatal day 4, EphA5 mRNA expression in retinal ganglion cells was found to be significantly $(p<0.05)$ elevated in hmz GNR23 mice, although the normal nasotemporal gradient of expression (Brown et al., 2000 ) was preserved ( $n=5$, each genotype; data not shown). Hemizygous GNR23 mice displayed the same pattern of upregulation, but with levels of EphA5 mRNA that were intermediate between those of wild-type and hmz GNR23 embryos (data not shown). The same elevated patterns of EphA5 mRNA expression were detected in younger GNR23 E11.5 and E12.5 mice. Oligonucleotide probes hybridizing to the extracellular Ig, transmembrane, or kinase coding domains of EphA5 mRNA gave identical results, suggesting that full-length EphA5 mRNA was being transcribed in GNR23 mice. Immunostaining for EphA5 in E14.5 GNR23 mice demonstrated increased EphA5 immunoreactivity in the same regions as those found to express elevated EphA5 mRNA (Fig. 4C,D), indicating that increased EphA5 mRNA was translated.

In the nose, negligible to very low levels of EphA5 mRNA were detected in the developing vomeronasal organ and lateral olfactory placode of wild-type E11.5 embryos (Fig. 5A). However, this was increased ( $p<0.05 ; n=4$ each genotype) approximately twofold in hmz GNR23 mice (Fig. 5B). At E14.5, EphA5 mRNA was no longer highly expressed in the vomeronasal organ but was found within the nasal mesenchyme through which GnRH neurons migrate (Fig. 5C, arrowheads). At E12.5, EphA5 protein was detected by tyramide-amplified immunofluorescence within clusters of cells adjacent to and nearby the vomeronasal organ, and the staining of adjacent sections for GnRH showed that these represented the GnRH neuron spherical clusters (Fig. 5E,F). These data demonstrate that a transgene integration event resulting in the deletion of $26 \mathrm{~kb}$ of sequence $67 \mathrm{~kb} \mathrm{3}$ to the Epha5 coding region has profound, brain region-specific effects on EphA5 expression in the developing brain. The GnRH neurons appear to overexpress EphA5 from their time of birth within the developing vomeronasal organ, and this continues as they attempt to migrate out of the vomeronasal organ and aggregate together to form atypical spherical clusters in the surrounding nasal mesenchyme.
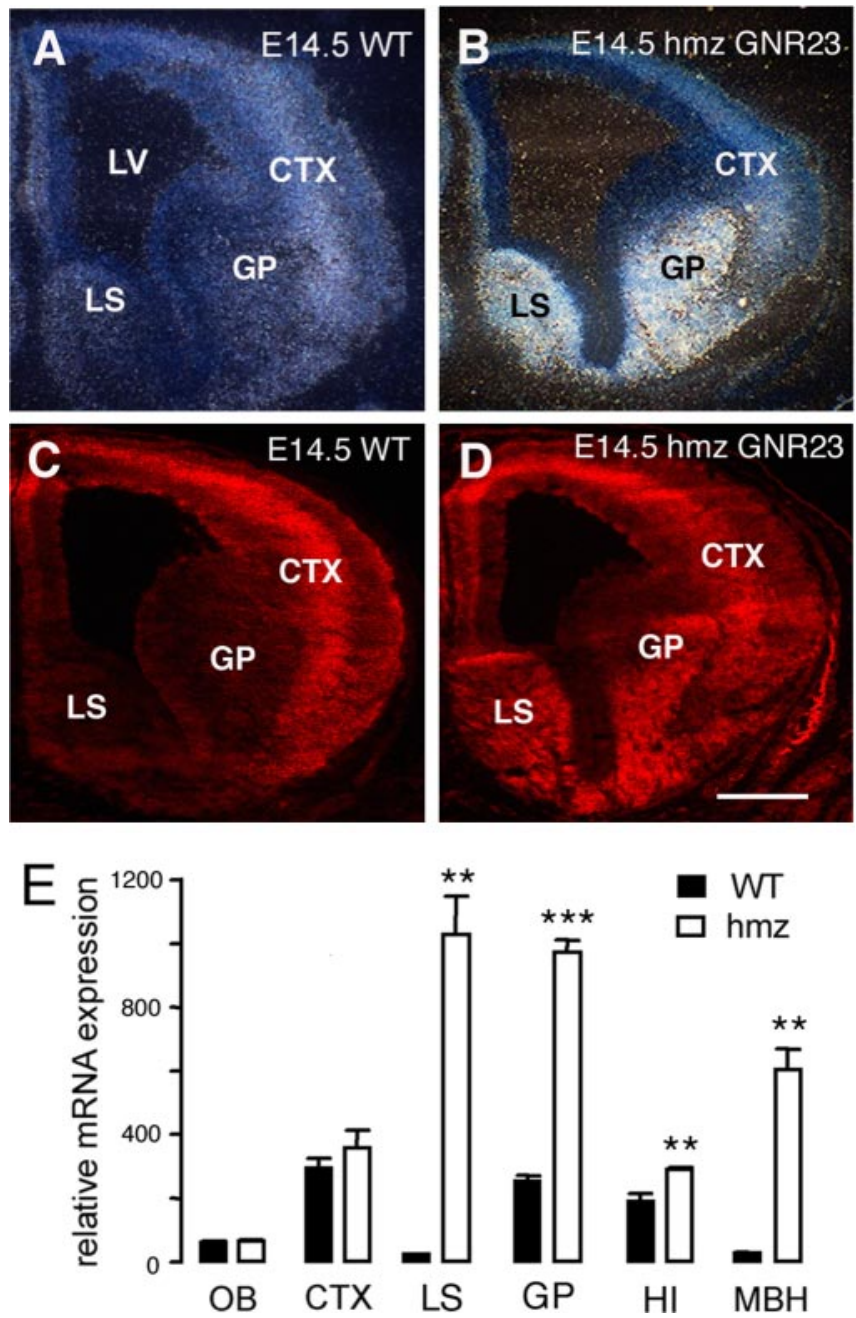

WT

$\mathrm{hmz}$

Figure 4. Levels of EphA5 mRNA and protein are upregulated in a brain region-specific manner in embryonic GNR23 mice. $A$, Dark-field coronal view of a wild-type E14.5 embryo brain showing differential distribution of EphA5 mRNA after radioactive in situ hybridization. $\boldsymbol{B}$, Same coronal level in an E14.5 hmz GNR23 littermate showing massive upregulation of EphA5 mRNA expression in the lateral septum (LS) and developing globus pallidus (GP) but not in the neocortex (CTX). C, D, EphA5 immunoreactivity in an E14.5 wild-type (C) and an hmz GNR23 (D) embryo showing upregulation of immunostaining in the same areas found for EphA5 mRNA. $\boldsymbol{E}$, Quantitative analysis of EphA5 mRNA expression within the olfactory bulb (OB), CTX, LS, GP, hippocampus (HI), and mediobasal hypothalamus (MBH) of wild-type (WT) and hmz GNR23 E14.5 embryos ( $n=4$ per group) after radioactive in situ hybridization. LV, Lateral ventricle. ${ }^{* *} p<0.01,{ }^{* * *} p<0.001$ compared with WT measurement. Scale bar, $600 \mu \mathrm{m}$.

\section{Ephrin-A mRNA expression}

Previous studies have indicated that vomeronasal organ and olfactory axons express ephrin A3 and A5 from E12.5 onward in the mouse (Knoll et al., 2001). To evaluate the ephrins being expressed by vomeronasal organ axons at E11.5, when the GnRH migratory process is first disrupted, and to assess any potential compensatory changes in GNR23 embryos, we undertook in situ hybridizations for ephrin A3 and A5 mRNAs in E11.5 and E12.5 wild-type and GNR23 embryos. At E11.5, only ephrin A3 mRNA was detectable in the vomeronasal organ (Fig. 5D). Ephrin A3 mRNA was also detected in the developing olfactory epithelium around the lateral olfactory placode (Fig. $5 D$ ). In agreement with Knoll et al. (2001), both ephrin A3 and A5 mRNAs were found in abundance within the vomeronasal organ at E12.5. The levels of ephrin A3 and A5 mRNA expression were not different between wild-type and hmz GNR23 embryos (data not shown). 


\section{Discussion}

We report here that the random integration of an unexpressed GnRH-related transgene has resulted in a mouse line where $\mathrm{GnRH}$ neurons fail to migrate normally into the brain. Phenotypic characterization of the GNR23 line shows that most embryonic GnRH neurons are slow to leave the developing vomeronasal organ and then fail to migrate normally along the vomeronasal axons to reach the brain. The few GnRH neurons that do enter the brain then appear to prefer to populate the MS, the brain region requiring the shortest migratory journey. Positional cloning strategies demonstrated that integration of the transgene resulted in the deletion of a $25.7 \mathrm{~kb}$ fragment of chromosome 5 that is required for correct regulation of the Epha5 gene. The consequent upregulation of EphA5 expression in developing GnRH neurons correlates with their marked migratory defects and indicates that disrupted ephrin signaling is not compatible with normal GnRH neuron migration along the vomeronasal axons.

An understanding of the precise mechanisms underlying the slowed migration and clumping of the GnRH neurons in GNR23 mice will require additional investigation. In addition to repulsive actions, adhesive and attractive properties of ephrin signaling have been uncovered in the process of neuronal development (Wilkinson, 2001; Holmberg and Frisen, 2002). As such, the GnRH neuron migratory defects may arise from enhanced EphA5-ephrin-dependent adhesiveness between the GnRH neuron and the vomeronasal organ axon, or increased repulsion between the GnRH neuron and an ephrin chemical gradient within the nose. In terms of this latter mechanism, it is important to note that although most GnRH neuron clusters were found near their migration start site, others could be identified as far away as the junction between the nose and brain. This suggests that any repulsive ephrin gradient that may be responsible for the $\mathrm{GnRH}$ neuron phenotype has no tight spatial definition within the nose. It is unlikely that the GnRH phenotype results from a tyrosine kinasetruncated EphA5 acting in a dominant-negative manner (Holmberg et al., 2000), because our in situ probe directed to the tyrosine kinase coding region of EphA5 mRNA also showed massive upregulation in GNR23 mice.

In support of a mechanism involving abnormal GnRH neuron-vomeronasal organ axon adhesiveness, ephrin A3 and ephrin A5 are known to be present along the full extent of the olfactory axons during development (Zhang et al., 1996; Knoll et al., 2001; St. John et al., 2002), and we show that ephrin A3 mRNA is expressed as early as E11.5 in the vomeronasal organ when the GnRH neurons commence their migration. Thus, it is possible that EphA5-expressing GnRH neurons interact directly with ephrin A3/A5 in their axophilic migration along the vomeronasal organ axons and that inappropriate EphA5 clustering and/or signal activation in $\mathrm{GnRH}$ neurons increases adhesiveness and disrupts this process. This possibility would be compatible with the slowed migratory rate detected. The mechanisms through which ephrins generate adhesive interactions are presently unclear
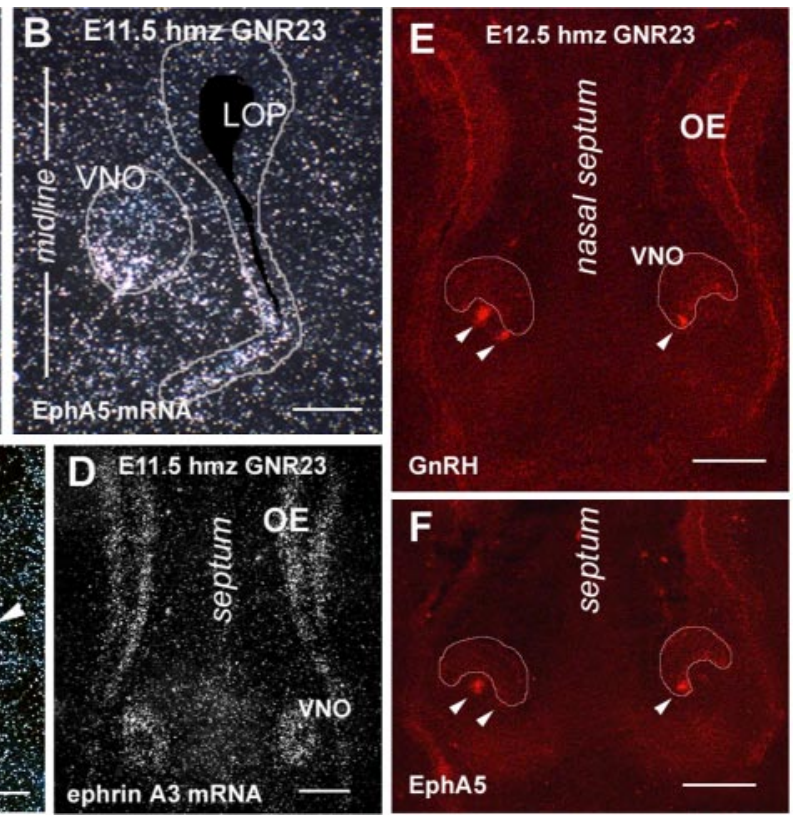

EphA5

Figure 5. Overexpression of EphA5 in nose and migrating $G n R H$ neurons in GNR23 mice. $A, B$, Coronal views of developing nose (WT; $\boldsymbol{A}$ ) and hmz GNR23 (B) embryos after EphA5 mRNA in situ hybridization. Note the increased silver grain

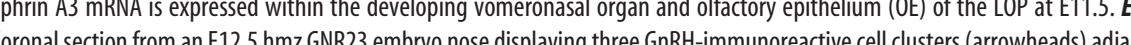
cent to the vomeronasal organ (outlined). Staining of the next consecutive section for EphA5 $(\boldsymbol{F})$ demonstrates that these spherical clusters are also expressing EphA5 (arrowheads). Scale bars: $\boldsymbol{A}, \boldsymbol{B}, \boldsymbol{E}, \boldsymbol{F}, 50 \mu \mathrm{m} ; \boldsymbol{C}, \boldsymbol{D}, 100 \mu \mathrm{m}$.

(Wilkinson, 2001). Roles for the ephexin-dependent modulation of Rho GTPase activity controlling polymerization of the actin cytoskeleton (Shamah et al., 2001) and the modulation of integrin-mediated adhesion by focal adhesion and other kinases (Miao et al., 2000) have been proposed. In addition, in the presence of increased EphA5-ephrin A3/A5 binding, there may be insufficient amounts of the metalloprotease kuzbanian to cleave the Eph-ephrin complexes and allow movement (Hattori et al., 2000). One clue to the pathway involved here, however, may be the observation of robust PSA-NCAM expression by GnRH neurons caught within clusters in GNR23 mice. Under normal circumstances, only a very few migrating GnRH neurons express PSA-NCAM (Yoshida et al., 1999), and the high level of PSANCAM immunoreactivity suggests upregulated PSA-NCAM expression by the clustered GnRH neurons. Thus, increased EphA5 expression in $\mathrm{GnRH}$ neurons may induce the expression of classical adhesion molecules as a consequence of upregulated Ephephrin signaling.

Relatively little is known about the organization and regulation of the EphA5 gene. The gene contains $\sim 17$ exons spanning $360 \mathrm{~kb}$ and, to our knowledge, there have been no evaluations of its promoter or flanking regulatory sequences. We show here that important sequences exist in the $3^{\prime}$ flank of the gene that are $>420 \mathrm{~kb}$ distant from its transcriptional start site. Expression of EphA5 in GNR23 mice was elevated in many brain regions such as the lateral septum, globus pallidus, and hippocampus but not in others, notably the cortex. This indicates that the $26 \mathrm{~kb}$ of sequence deleted in GNR23 mice is likely to contain elements that are used by neurons in a heterogeneous manner to repress Epha5 gene expression. As might be expected, these observations indicate that the mechanisms of EphA5 gene regulation will be com- 
plex and cell dependent. Although no other genes appear to exist within $500 \mathrm{~kb}$ either side of the $26 \mathrm{~kb}$ deletion, it remains possible that sequences within the deleted area may also be important in the regulation of more distant genes on chromosome 5. One of these is the GnRH receptor, but our in vivo studies in adult GNR23 mice (our unpublished data) indicate that this receptor functions normally in the mutant mice. It is noteworthy, however, that a clear allele- and dose-response relationship existed between the degree of EphA5 upregulation and GnRH neuron migratory disruption in hemizygous and hmz mice.

Intriguingly, $\sim 15 \%$ of the GnRH neurons in hmz GNR23 embryos appear to migrate relatively normally out of the nose but then prefer to populate regions requiring the shortest migratory distance within the brain. Why this small population of $\mathrm{GnRH}$ neurons is relatively unaffected by the Epha5 gene disruption is unknown. Because both the level of EphA5 upregulation and numbers of $\mathrm{GnRH}$ neurons reaching the brain are clearly allele dependent, it seems most likely that "escaping" GnRH neurons do not overexpress EphA5 sufficiently to inhibit their migration. Alternatively, these cells may not be unaffected by the EphA5 upregulation and may instead represent GnRH neurons that would have migrated farther caudal under normal circumstances. There is evidence for marked heterogeneity in the properties and functioning of the GnRH neuronal phenotype, and at least some of this appears to exist from the time of birth (Tobet et al., 2001; Wray, 2002). Hence, these cells may represent a functional subtype of the GnRH neurons. Importantly, we find that these GnRH neurons are not sufficient on their own to enable normal fertility in hmz adult female GNR23 mice. Recently, it was reported that EphA $5^{\text {lacZ/lacZ }}$ mice with a functional tyrosine kinase deletion in the Epha5 gene were fertile (Feldheim et al., 2004). Although an analysis of the GnRH neurons has not been undertaken in those mice, this suggests that EphA5 tyrosine kinase-dependent signaling is dispensable or replaceable in terms of GnRH neuron migration.

In summary, these data reveal that normal levels of ephrin signaling are critical for $\mathrm{GnRH}$ neuron axophilic migration, with an upregulation in EphA5-ephrin signaling in the GnRH neuronal phenotype resulting in slowed migration and clustering. $\mathrm{Mu}-$ tations in both $K A L-1$ and fibroblastic growth factor receptor-1 have been identified to result in infertility in humans (Hardelin, 2001; Dode et al., 2003). In the case of $K A L-1$, it is thought that defects in the encoded protein anosmin-1 may result in the failure of olfactory nerve axons to branch correctly and penetrate the cribriform plate and, consequently, the failure of GnRH neurons to migrate into the brain (MacColl et al., 2002b). The dependence of GnRH migration, and thus fertility, on normal levels of ephrin signaling reported here suggests that defects in ephrin-related molecules may represent an additional class of genes underlying hypogonadotropic hypogonadism in humans.

\section{References}

Brown A, Yates PA, Burrola P, Ortuno D, Vaidya A, Jessell TM, Pfaff SL, O'Leary DD, Lemke G (2000) Topographic mapping from the retina to the midbrain is controlled by relative but not absolute levels of EphA receptor signaling. Cell 102:77-88.

Dode C, Levilliers J, Dupont JM, De Paepe A, Le Du N, Soussi-Yanicostas N, Coimbra RS, Delmaghani S, Compain-Nouaille S, Baverel F, Pecheux C, Le Tessier D, Cruaud C, Delpech M, Speleman F, Vermeulen S, Amalfitano A, Bachelot Y, Bouchard P, Cabrol S, et al. (2003) Loss-offunction mutations in FGFR1 cause autosomal dominant Kallmann syndrome. Nat Genet 33:463-465.

Feldheim DA, Nakamoto M, Osterfield M, Gale NW, DeChiara TM, Rohatgi R, Yancopoulos GD, Flanagan JG (2004) Loss-of-function analysis of EphA receptors in retinotectal mapping. J Neurosci 24:2542-2550.
Flanagan JG, Vanderhaeghen P (1998) The ephrins and Eph receptors in neural development. Annu Rev Neurosci 21:309-345.

Franklin KBJ, Paxinos G (1997) The mouse brain in stereotaxic coordinates. San Diego: Academic.

Frisen J, Holmberg J, Barbacid M (1999) Ephrins and their Eph receptors: multitalented directors of embryonic development. EMBO J 18:5159-5165.

Gale NW, Holland SJ, Valenzuela DM, Flenniken A, Pan L, Ryan TE, Henkemeyer M, Strebhardt K, Hirai H, Wilkinson DG, Pawson T, Davis S, Yancopoulos GD (1996) Eph receptors and ligands comprise two major specificity subclasses and are reciprocally compartmentalized during embryogenesis. Neuron 17:9-19.

Hansen MJ, Dallal GE, Flanagan JG (2004) Retinal axon response to ephrin-as shows a graded, concentration-dependent transition from growth promotion to inhibition. Neuron 42:717-730.

Hardelin JP (2001) Kallmann syndrome: towards molecular pathogenesis. Mol Cell Endocrinol 179:75-81.

Hattori M, Osterfield M, Flanagan JG (2000) Regulated cleavage of a contact-mediated axon repellent. Science 289:1360-1365.

Holland SJ, Gale NW, Mbamalu G, Yancopoulos GD, Henkemeyer M, Pawson T (1996) Bidirectional signalling through the EPH-family receptor Nuk and its transmembrane ligands. Nature 383:722-725.

Holmberg J, Frisen J (2002) Ephrins are not only unattractive. Trends Neurosci 25:239-243.

Holmberg J, Clarke DL, Frisen J (2000) Regulation of repulsion versus adhesion by different splice forms of an Eph receptor. Nature 408:203-206.

Isles AR, Ma D, Milsom C, Skynner MJ, Cui W, Clark J, Keverne EB, Allen ND (2001) Conditional ablation of neurones in transgenic mice. J Neurobiol 47:183-193.

Knoll B, Drescher U (2002) Ephrin-As as receptors in topographic projections. Trends Neurosci 25:145-149.

Knoll B, Zarbalis K, Wurst W, Drescher U (2001) A role for the EphA family in the topographic targeting of vomeronasal axons. Development 128:895-906.

Kullander K, Klein R (2002) Mechanisms and functions of Eph and ephrin signalling. Nat Rev Mol Cell Biol 3:475-486.

MacColl G, Quinton R, Bouloux PM (2002a) GnRH neuronal development: insights into hypogonadotrophic hypogonadism. Trends Endocrinol Metab 13:112-118.

MacColl G, Bouloux P, Quinton R (2002b) Kallmann syndrome: adhesion, afferents, and anosmia. Neuron 34:675-678.

Miao H, Burnett E, Kinch M, Simon E, Wang B (2000) Activation of EphA2 kinase suppresses integrin function and causes focal-adhesion-kinase dephosphorylation. Nat Cell Biol 2:62-69.

Pape J-R, Skynner MJ, Allen ND, Herbison AE (1999) Transgenics identify distal $5^{\prime}$ and $3^{\prime}$ sequences specifying gonadotropin-releasing hormone expression in adult mice. Mol Endocrinol 13:2203-2211.

Rowe LB, Nadeau JH, Turner R, Frankel WN, Letts VA, Eppig JT, Ko MS, Thurston SJ, Birkenmeier EH (1994) Maps from two interspecific backcross DNA panels available as a community genetic mapping resource. Mamm Genome 5:253-274.

Schwanzel-Fukuda M, Pfaff DW (1989) Origin of luteinizing hormonereleasing neurons. Nature 338:161-164.

Schwanzel-Fukuda M, Abraham S, Crossin KL, Edelman GM, Pfaff DW (1992) Immunocytochemical demonstration of neural cell adhesion molecule (NCAM) along the migration route of luteinizing hormonereleasing hormone (LHRH) neurons in mice. J Comp Neurol 321:1-18.

Shamah SM, Lin MZ, Goldberg JL, Estrach S, Sahin M, Hu L, Bazalakova M, Neve RL, Corfas G, Debant A, Greenberg ME (2001) EphA receptors regulate growth cone dynamics through the novel guanine nucleotide exchange factor ephexin. Cell 105:233-244.

Simonian SX, Herbison AE (2001a) Differing, spatially restricted roles of ionotropic glutamate receptors in regulating the migration of $\mathrm{GnRH}$ neurons during embryogenesis. J Neurosci 21:934-943.

Simonian SX, Herbison AE (2001b) Regulation of gonadotropin-releasing hormone $(\mathrm{GnRH})$ gene expression during $\mathrm{GnRH}$ neuron migration in the mouse. Neuroendocrinology 73:149-156.

Skynner MJ, Slater R, Sim JA, Allen ND, Herbison AE (1999) Promoter transgenics reveal multiple gonadotropin-releasing hormone-1-expressing cell populations of different embryological origin in mouse brain. J Neurosci 19:5955-5966.

St John JA, Pasquale EB, Key B (2002) EphA receptors and ephrin-A ligand- 
sexhibit highly regulated spatial and temporal expression patterns in the developing olfactory system. Brain Res Dev Brain Res 138:1-14.

Tobet SA, Bless EP, Schwarting GA (2001) Developmental aspect of the gonadotropin-releasing hormone system. Mol Cell Endocrinol 185: 173-184.

Weinl C, Drescher U, Lang S, Bonhoeffer F, Loschinger J (2003) On the turning of Xenopus retinal axons induced by ephrin-A5. Development 130:1635-1643.

Wilkinson DG (2001) Multiple roles of EPH receptors and ephrins in neural development. Nat Rev Neurosci 2:155-164.

Wray S (2002) Development of gonadotropin-releasing hormone-1 neurons. Front Neuroendocrinol 23:292-316.
Wray S, Grant P, Gainer H (1989) Evidence that cells expressing luteinizing hormone-releasing hormone mRNA in the mouse are derived from progenitor cells in the olfactory placode. Proc Natl Acad Sci USA 86:8132-8136.

Wray S, Key S, Qualls R, Fueshko SM (1994) A subset of peripherin positive olfactory axons delineates the luteinizing hormone releasing hormone neuronal migratory pathway in developing mouse. Dev Biol 166:349-354.

Yoshida K, Rutishauser U, Crandall JE, Schwarting GA (1999) Polysialic acid facilitates migration of luteinizing hormone-releasing hormone neurons on vomeronasal axons. J Neurosci 19:794-801.

Zhang JH, Cerretti DP, Yu T, Flanagan JG, Zhou R (1996) Detection of ligands in regions anatomically connected to neurons expressing the Eph receptor Bsk: potential roles in neuron-target interaction. J Neurosci 16:7182-7192. 